\title{
Óptimo despliegue y enrutamiento de UDAP para infraestructura de medición avanzada basada en el algoritmo MST
}

\author{
Optimal deployment and routing geographic of UDAP for advanced \\ metering infrastructure based on MST algorithm
}

\author{
Cristian Ganán ${ }^{1 *} \quad$ Esteban Inga $^{1} \quad$ Roberto Hincapié ${ }^{2}$
}

Recibido 3 de junio de 2015, aceptado 26 de mayo de 2016

Received: June 3, 2015 Accepted: May 26, 2016

\begin{abstract}
RESUMEN
El presente artículo permite optimizar el costo por despliegue de los puntos de agregación de datos universales (UDAP) para la infraestructura avanzada de medición inteligente (AMI) considerando cobertura y capacidad. Se presenta un modelo de enrutamiento entre UDAPs basado en minimum spanning tree (MST) para encontrar el mínimo número de enlaces en una zona georreferenciada específica. Al estar georreferenciada se logra que la localización de UDAP y la topología de la red sean reales. La búsqueda de los lugares óptimos de un conjunto de sitios candidatos advierte la necesidad de un algoritmo de optimización que permita minimizar los sitios candidatos y los convierta en sitios factibles activos en el diseño previo al despliegue de la red. Los resultados obtenidos implican un punto base firme para que las empresas de distribución eléctrica establezcan el número adecuado de concentradores (UDAP). Dentro del trabajo se consideran ciertas tecnologías de comunicación aplicadas en AMI como: redes celulares (CDMA, GPRS, LTE) o el estándar IEEE 802.15.4 g. El menor costo basado en el uso óptimo de recursos para despliegue de infraestructura toma gran importancia para la planificación y escalabilidad de la red de AMI.
\end{abstract}

Palabras clave: Redes eléctricas inteligentes, infraestructura de medición avanzada, punto de agregación de datos universal, mínimo árbol de expansión, optimización, costos, red de sensores inalámbricos.

\section{ABSTRACT}

This paper optimizes the cost of deployment of universal data aggregation points (UDAP) for advanced smart metering infrastructure (AMI), considering coverage and capacity. We propose a routing model between UDAPs, based on minimum spanning tree algorithm (MST) to find the minimum number of links in a specific geo-referenced area. Because it is geo-referenced, the location of the UDAP and the topology of the net are real. The search for optimal locations of a set of candidate sites warns the necessity of an optimization algorithm to minimize candidate sites and turn them into active feasible sites in the previous design to the network deployment. Results imply a strong base point for electricity distribution companies to establish the right number of concentrators (UDAP). There are certain applied AMI communication technologies considered, such as: cellular networks (CDMA, GPRS, and LTE) or the IEEE 802.15.4 g standard. The lowest cost based on the optimal resources usage in infrastructure deployment is very important for planning and scalability phases of AMI network.

Keywords: Smart grid, advanced metering infrastructure, universal data aggregation point, minimum spanning tree, optimization, cost, wireless sensor networks.

1 Departamento de Ingeniería Eléctrica. Universidad Politécnica Salesiana. Quito, Ecuador.

E-mail: cganan@est.ups.edu.ec; einga@ups.edu.ec

2 Departamento de Ingeniería Electrónica. Universidad Pontificia Bolivariana. Medellín, Colombia.

E-mail: roberto.hincapie@upb.edu.co

* Autor de correspondencia 


\section{INTRODUCCIÓN}

La infraestructura de medición inteligente (AMI), por sus siglas en inglés, es una aplicación de los redes de sensores inalámbricas (WSN) que incluyen sensores o medidores inteligentes (SM), sistemas de comunicación variados que permiten el monitoreo, control y cobro del uso de diferentes tipos de servicios: electricidad, gas de uso doméstico, agua potable y otros, siendo una de las partes más críticas el despliegue de la red inalámbrica empleada para la conectividad de dichos SM [1-3].

El uso de AMI implica grandes ventajas: lectura remota (enlace ascendente - SM empresa distribuidora), desconexión y reconexión automática (enlace descendente - empresa distribuidora - SM), rápida reposición de servicio en contingencias, implantación de servicio prepago lo que se traduce en una mejora significativa de la calidad del servicio a los usuarios finales. Así para garantizar la recolección de información se deben usar recursos de manera óptima con el fin de minimizar los costos. De esta forma reutilizar tecnologías permite reducir los costos de una nueva inversión entre las que podemos incluir redes celulares y otras de menor alcance enfocadas a redes inteligentes como IEEE802.15.4 g, en oposición de las tecnologías cableadas que por las limitaciones de alcance y la complejidad de implementación en zonas de difícil acceso y rurales resultan poco prácticas, para su uso como infraestructura de comunicación de AMI en zonas rurales que es uno de los objetivos del presente trabajo [2, 4-5].

Por lo tanto el trabajo propone colocar un solo concentrador multirradio denominado UDAP o punto de agregación de datos universal, el mismo que permitirá cohabitar a las diferentes posibilidades en lo referente a tecnología de red inalámbrica.

Para el presente estudio se han planteado tres etapas que garanticen el objetivo de minimizar los costos relacionados con los recursos empleados, la primera se enmarca en la cobertura de poblaciones, la segunda etapa se relaciona al problema de capacidad de los concentradores y la última etapa consiste en el enrutamiento de la topología de red por medio de un mínimo árbol de expansión entre UDAP), con la que será posible hacer estructura multisalto de comunicación hasta llegar a la estación base más cercana [4-5].
Las metodologías que han sido empleadas previamente para la resolución del problema de la colocación óptima de diversos dispositivos de concentración tomando en cuenta poder cubrir a varios clientes en simultáneo; es decir, la primera etapa, incluyen la clusterización por medio de algoritmos como Dijkstra buscando la ruta más corta para los enlaces sin que la capacidad esté involucrada; K-Means, una alternativa más efectiva y menos compleja para la implantación en la que se pueden obtener agrupaciones más homogéneas con dispositivos diferentes; otros sistemas proponen la clusterización basada en la eficiencia energética (EECS), limitando la cantidad de dispositivos que se pueden colocar según una estimación sin que la escalabilidad sea óptima. Por otro lado, la colocación de puntos de agregación o de concentración se puede llevar a cabo mediante heurísticas: el algoritmo de Greedy que busca las soluciones cercanas a la óptima pero ha presentado problemas con grandes poblaciones o que se encuentren dispersas; como las poblaciones rurales en estudio [6-8].

Para la resolución de las siguientes etapas del problema planteado, los algoritmos previamente empleados por otros autores, están relacionados a un problema de optimización bajo programación lineal que permite introducir la función objetivo y sus restricciones resueltas en un escenario MILP para lograr minimizar los recursos y así disminuir los costos en el despliegue. Son varias las restricciones anteriormente planteadas para minimizar el número de UDAP de un conjunto de sitios candidatos, relacionadas con la capacidad de usuarios del concentrador bajo el mismo entorno, la distancia que puede cubrir el sistema de concentración, las condiciones de terreno y más limitaciones técnico-operativas propias de la naturaleza de una red inalámbrica y las tecnologías para que AMI sea factible.

En una siguiente etapa, al tener ubicados a los UDAP en la región a ser cubierta se requiere el mapa de enrutamiento para dichos concentradores por lo que se recurre a proponer un mínimo árbol de expansión de la topología de la red.

El algoritmo del árbol de mínima expansión o minimum spanning tree (MST) está basado en la teoría de grafos y puede ser resuelto bajo diversos algoritmos incluyendo el de Prim o el de Kruskal 


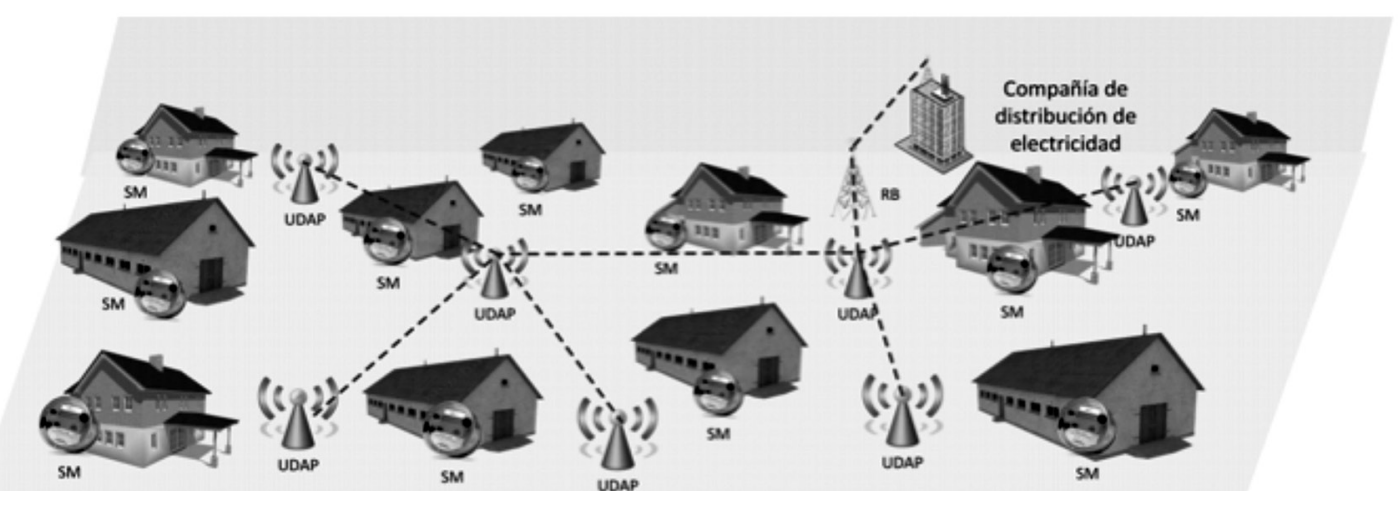

Figura 1. Infraestructura de medición inteligente, enrutamiento y dimensionamiento.

y demás que han mostrado en otros trabajos poder hallar la ruta o camino con el que se logra cubrir toda la demanda seleccionando la menor cantidad de dispositivos de entre los que ha sido dispuestos como candidatos logrando la optimización del costo $[5,8]$.

La red de AMI a partir de la colocación óptima de los UDAPs plantea escenarios con diversas cantidades de sensores (SM) y con capacidad diferentes respecto de tecnología inalámbrica dependiendo de las necesidades de cada sector urbano o rural, además de su topología de malla.

La metodología de Prim para generar el árbol de expansión se basa en la toma de cada uno de los nodos para interconectar a los demás puntos UDAPs, de tal forma de que se interconecten todos con los mínimos pesos de enlaces logrando que el árbol recorra la mínima distancia para llegar al punto de conexión hacia la gestión de los datos de la demanda de la compañía distribuidora de electricidad [9-10].

La capacidad de cobertura de un punto de agregación tiene una forma específica omnidireccional por lo que si se colocan en posiciones clave permitirán la recolección de datos eficientemente; por ello la facilidad que ofrecen los sistemas de posicionamiento global e información geográfica (GIS) mejorará la precisión para la implantación suponiendo un avance en las técnicas de optimización haciendo además a la presente propuesta universal $[5,11]$.

Este trabajo plantea un algoritmo efectivo en el que en un primer instante minimice la cantidad de puntos de agregación dentro de un escenario georreferenciado basado en sitios candidatos, considerando las limitaciones de cobertura, capacidad de las tecnologías empleadas para la implantación de una infraestructura de medición de energía eléctrica, de tal forma que cada medidor al menos pueda conectarse con un UDAP. En un segundo instante se busca que todos los concentradores se conecten entre sí para el envío de datos hacia la compañía de distribución, esto mediante la teoría de grafos y el MST con heurísticas que permiten la obtención de la alternativa más cercana a la óptima, con lo que se minimiza la cantidad de recursos necesarios para realizar la conexión. De esta forma, para próximos trabajos se podrá analizar el impacto económico en la implantación de un sistema de estas características más fácilmente bajo un esquema georreferenciado simulado, que puede ser aplicable en cualquier lugar y condición; pudiendo con las herramientas adecuadas manejar otros aspectos de las comunicaciones como latencias, limitaciones de banda y otros que hacen la implantación más compleja.

En adelante este artículo se organiza de la siguiente manera. En la sección II se introduce la relación del enrutamiento por medio del árbol de mínima expansión con la cobertura de poblaciones de medidores inteligentes según su capacidad. En la sección III se describe la formulación del problema de optimización para desplegar infraestructura en AMI. En la sección IV se disponen los resultados de la optimización de colocación de UDAP georreferenciados. Finalmente se concluye en la sección V. 


\section{DIMENSIONAMIENTO Y ENRUTAMIENTO DE LA RED DE SENSORES INALÁMBRICOS}

Para que un sistema AMI sea escalable en su diseño, debe ser flexible en tecnología, permitiendo así la inclusión de distintos tipos de infraestructuras de comunicación para el envío y recepción de la información. Las características de la medición inteligente advierte que los sensores-medidores inteligentes son fijos y, por tanto, la colocación de sitios de concentración y agregación de datos resulta más simple; sin embargo, se debe garantizar su cobertura, con limitantes en la capacidad de cada tecnología que pudiera ser empleada [11-12].

\section{Cobertura y capacidad de poblaciones georreferenciadas de medidores inteligentes}

Los medidores inteligentes deben ser considerados en red de sensores inalámbricos -wireless sensor networks (WSN)- y en tales redes uno de los problemas principales es el considerar la cobertura y la capacidad de la tecnología como uno solo. Lo más común en este sentido es que hayan enlaces multihop y en este caso se plantea la ocupación de redes distribuidas [7, 13-14].

Cada concentrador UDAP deberá tomar los datos de varios medidores de tal forma de que los enlaces sean óptimos y no se deban recorrer distancias excesivamente largas; entendiendo que la cantidad de información o tráfico generado y las condiciones del canal de datos serán condiciones que también modifiquen el tratamiento del problema pero están fuera del alcance de este trabajo. Uno de los principales planteamientos es el uso de ubicaciones georreferenciadas para cada uno de los equipamientos que se vaya a colocar, por lo tanto la fórmula de Haversine es de gran utilidad para calcular la distancia entre dos puntos georreferenciados. Se ha planteado un radio de cobertura $R$ de cada UDAP $\mathrm{y}$ una capacidad $\mathrm{C}$ de albergar hasta $\mathrm{N}$ usuarios (medidores inteligentes) y un número de $\mathrm{M}$ sitios factibles donde pueden ser colocados los UDAP $[6,10,15-16]$.

El problema de la cobertura que se ha establecido corresponde a que cada SM debe ser cubierto por al menos un UDAP dependiente directamente de la distancia que abarca el punto de agregación, en este caso lo que se busca es atender al SM que se encuentra en la zona de cobertura del UDAP [16-17].

Por otro lado, para resolver el problema planteado se lo realiza por medio de programación lineal (MILP) con el objeto de minimizar el número de UDAP que satisfagan la cobertura deseada y de esta manera minimizar el costo por los recursos al ser desplegados $[2,17]$, a esto se suma la posibilidad de incluir una restricción que limite la capacidad de albergar un número definido de SM en cada UDAP, condicionante que de igual manera se escribe mediante el uso de Matlab sobre LPSolve que es el software que permite resolver el problema de optimización y su resultado optimizado es leído nuevamente desde Matlab para obtener la gráfica georreferenciada de la zona de despliegue con el resultado óptimo para el despliegue de los puntos de agregación UDAP.

\section{Árbol de expansión con redes de sensores}

Para la resolución del problema de enrutamiento, en este trabajo se plantea un árbol de mínimo costo para interconexión de UDAP que se resuelve por medio de una heurística MST que se basa en el uso del algoritmo de Prim. Lo mencionado permite que la comunicación se puede llevar a cabo entre todos los puntos de uno a otro; minimizando la cantidad de recursos necesarios, objetivo del presente trabajo.

Dentro del planteamiento del MST se advierte al primer paso que corresponde a la formación de un grafo $\mathrm{G}\left(V, E, W_{i, j}\right)$ que contenga todas las conexiones $E$ entre el $i$-ésimo y el j-ésimo pares de vértices $V$ que en el estudio representan a los UDAPs. Cada conexión tiene un peso específico $W_{i, j}$ que corresponde a la distancia que existe entre ellos [18-21].

Con esquemas como el de Prim y el de Kruskal se puede resolver u obtener el que resulta la ruta más óptima para la interconexión minimizando la distancia representada por lo que a la vez minimiza el costo representado una razón por la que debe cruzarse para llegar hacia la estación base y posteriormente al centro de gestión de los datos de la demanda eléctrica, siendo este camino la columna vertebral de la arquitectura distribuida de comunicación $[6,22]$. 
La evolución del sistema de Prim según $[9,23]$ es la siguiente:

Entradas: Un grafo que no está vacío con los nodos $V$ y las aristas o conexiones $E$

Inicializar: Para todo $i \in V$

$$
\text { Haga } V_{\text {nuevo }}=\{i\}
$$

Escoger: Arista $i, j$ con $W_{i, j}$ que sea mínimo tal que $i$ sea $V_{\text {nuevo }}$ y no.

Principal: Añadir $j$ a $V_{\text {nuevo }}(i, j)$ a $E_{\text {nuevo; }}$ formando el MST.

Las redes de sensores son la clave para la recolección de datos en ambientes alejados u hostiles; siendo la cobertura uno de los problemas más fundamentales y una medida de la calidad del monitoreo que se le da a la región y de la calidad de servicio. El dimensionamiento de la red deberá ser llevado a cabo de tal forma que cada medidor sea cubierto por al menos un punto de agregación siendo dependiente en forma directa del rango de cobertura que posea el UDAP, así como de la cantidad de usuarios o capacidad que tengan los puntos de agregación $[13,24]$.

Para el caso específico de la medición inteligente los medidores contarán con ubicaciones específicas por lo que la redundancia de las comunicaciones así como el consumo de energía no serán puntos tan críticos a tomar en consideración.

\section{FORMULACIÓN DEL PROBLEMA}

El problema más importante supone el maximizar la cobertura mediante la colocación óptima de los puntos de agregación en el área cubierta, recordando que la implantación de este sistema deberá considerar posteriormente el tráfico y las condiciones del canal funcionando con SM. Para esto se considerarán promedios de cobertura de las tecnologías inalámbricas más usadas $R=\left\{R_{j}\right\}_{j=1, \ldots ., M}$ y una capacidad del número de usuarios $C=\left\{C_{j}\right\}_{j=1, \ldots, M}$ [25-27], siendo $\mathrm{M}$ la cantidad de sitios candidatos para colocar UDAP planteados.

La optimización del número de puntos de agregación según las restricciones de la capacidad y de la cobertura se lleva a cabo mediante programación lineal entera mixta (MILP) considerando que del total de medidores o usuarios $N$ que existan al menos $P$ en porcentual usuarios son efectivamente cubiertos, siendo $Y_{i} \in\{0,1\}$, un valor que se le da a cada medidor para indicar con 1 si está efectivamente cubierto por al menos un sitio candidatos o 0 en caso de no estarlo, lo que se representa mediante la ecuación (1):

$$
\frac{1}{N} \sum_{i=1}^{N} Y_{i} \geq P
$$

Por otro lado, debe ser considerado que los problemas debido a interferencia o existencia de obstáculos naturales y artificiales están fuera del alcance del presente trabajo siendo la distancia el principal efecto a tomar en cuenta para el dimensionamiento de la red y los algoritmos que se han planteado en la sección.

Para el presente trabajo se ha considerado que existe un área A con límites georreferenciados específicos en la que deben ser cubiertos los medidores inteligentes $N$ mediante el emplazamiento de sitios candidatos de UDAPs $M$, que permiten la conectividad a usuarios dentro de un radio de cobertura de $R$ metros a kilómetros donde las distancias son calculadas mediante la fórmula de Haversine y vectores de latitud y longitud de medidores y sitios candidatos, representando este punto al dimensionamiento de la red, cada UDAP tiene una capacidad de usuarios $C$.

Se debe considerar que para el presente trabajo se usó de una red celular de tipo GPRS como base, en la que las estaciones analizadas tienen una cobertura de dos $\mathrm{km}$ en promedio y que cada una puede albergar hasta 300 SM denotando que el tráfico asociado será bajo si se toma en cuenta que la medición asociada no requiere de datos en tiempo real. Dentro de las restricciones más importantes se determina que los usuarios están en cualquier posición dentro de la región y los UDAPs se colocarán en la infraestructura ya existente de la compañía distribuidora de electricidad incluyendo postes exteriores. Se considera que un usuario está cubierto si se encuentra dentro de la distancia $R$ de al menos un UDAP. El problema puede ser resumido mediante:

\section{Función objetivo:}

$$
\min \sum_{j=1}^{M} Z_{j}
$$


Sujeto a:

$$
\begin{gathered}
Y_{i} \leq \sum_{j=1}^{M} \alpha_{i, j} \times Z_{j} ; \forall i \in U \forall j \in S \\
\sum_{i=1}^{N} \alpha_{i, j} \leq C \times Z_{j} ; \forall i \in U
\end{gathered}
$$

La ecuación (2) representa que se minimiza la cantidad de sitios candidatos, $Z_{j}$ representando a cada sitio candidato, si es un sitio factible vale 1 y 0 en caso contrario, la restricción (3) indica que si no hay un sitio candidato que cubra al usuario necesariamente $Y_{i}$ vale 0 , es decir el usuario no está cubierto; en este caso $\alpha_{i, j}$ que vale 1 si el usuario $i$ es cubierto por el UDAP $j$, de lo contrario el valor es 0 ; con un conjunto $U$ de usuarios seleccionados y un conjunto $S$ de sitios candidatos. Además se debe considerar la ecuación (1) cómo restricción. La restricción (4) implica que la cantidad de usuarios conectados a un sitio candidato activo no puede exceder la capacidad $C$.

Luego de determinar las distancias que son más cortas se logra que por MILP se minimice la cantidad de UDAPs que son colocados de acuerdo a los sitios candidatos factibles que finalmente son los activos. El pseudocódigo que permite dimensionar la red

\begin{tabular}{|c|c|}
\hline \multicolumn{2}{|r|}{$\begin{array}{l}\text { Algoritmo de optimización de } \\
\text { cobertura y capacidad de UDAP }\end{array}$} \\
\hline Paso 1: & Leer archivo SRTM con límites \\
\hline Paso 2: & Graficar sitios candidatos UDAPs, SM y EB \\
\hline \multirow{4}{*}{ Paso 3: } & Para todo: $i \in U$ y $j \in S$ \\
\hline & Si: $d_{i, j}<R$ \\
\hline & Haga: Sitio candidato $=1$ \\
\hline & Caso contrario: Sitio candidato $=0$ \\
\hline \multirow{4}{*}{ Paso 4: } & Para todo: $i \in U$ y $j \in S$ \\
\hline & Si Num. Usuarios en $j<=C$ y Sitio candidato $=1$ \\
\hline & Haga: Usuario $i \in \operatorname{UDAP} j$ \\
\hline & Caso contrario: Usuario $i$ no $\in \operatorname{UDAP} j$ \\
\hline Paso 5: & $\begin{array}{l}\text { Problema primario: encuentre Sitios candi- } \\
\text { datos óptimos. }\end{array}$ \\
\hline Paso 6: & $\begin{array}{l}\text { Graficar sitios candidatos elegidos y enlaces } \\
\text { SM-UDAP. }\end{array}$ \\
\hline \multicolumn{2}{|r|}{ Terminar } \\
\hline
\end{tabular}
es el siguiente:

La conectividad entre UDAPs es la segunda parte, en este caso se supone la existencia de un conjunto de nodos $V$ con $E$ como el conjunto de enlaces existentes entre pares de vértices, existiendo un grafo $G(V$, $E, W$ ) que representa la topología de la red. Para el presente trabajo lo que se ha planteado es el uso de una heurística de tal forma de obtener la solución más cercana a la óptima, lo que permitirá determinar cuál es el camino más adecuado para interconectar a todos los UDAPs que han sido elegidos entre sí.

Existirá un flujo de datos que requiere transmitir la información desde un nodo origen i-ésimo hasta un destino j-ésimo con un peso que puede ser la distancia u otro que resulte relevante, $W_{i, j}$, esto se representa mejor con las siguientes ecuaciones.

\section{Función objetivo:}

$$
\min \sum_{(i, j) \in E} W_{i, j} \times X_{i, j}
$$

\section{Sujeto a:}

$$
\begin{gathered}
\sum_{e_{i, j} \in E} X_{i, j}=K-1 \\
\sum_{e_{i=j} \in E} X_{i, j} \geq 1 ; \forall B \subset V, i \in B, j \in B
\end{gathered}
$$

Donde la (5) es el objetivo de minimizar la distancia que deba recorrerse de $i$ hacia $j$ según el peso $W_{i, j}$ y donde $X_{i, j}$ vale 1 si el enlace existe y pertenece al árbol $A$, mientras que tiene un valor de 0 en caso contrario. $B$ es un subconjunto cualquiera de nodos correspondiente, la resolución es llevada a cabo mediante la técnica de Prim antes explicada dependiendo de la cantidad de usuarios que deban ser albergados.

La restricción (6) implica que del total de nodos que pertenecen al escenario, la cantidad de enlaces $E$ que sean colocados será al menos $K-1$, siendo $K$ el número de nodos del sistema. La ecuación (7) indica que para realizar la optimización propuesta debe existir más de 1 enlace factible de tal forma de poder escoger el que tenga un peso minoritario.

Con esto se forma un árbol con un conjunto de enlaces $A=\left\{e_{1}, e_{2}, \ldots . ., e_{n}\right\}$ que tenga un costo mínimo y permita la llegada de la información hasta la estación base proveniente de los usuarios para ser transmitida a los centros de gestión de 
datos de la demanda, así como el paso multicast de la información; es decir, que la información se transmita desde cualquier punto de la red planteada hacia otro sin ningún problema, generando un tráfico que será muy bajo en relación al que se genera dentro de una red celular típica.

El pseudocódigo para la segunda parte se aprecia a continuación.

\begin{tabular}{|l|l|}
\hline \multicolumn{2}{|c|}{ Algoritmo de enrutamiento MST entre UDAP } \\
\hline Paso 1: & Leer archivo SRTM con límites \\
\hline Paso 2: & Graficar sitios candidatos elegidos UDAPs, SM. \\
\hline Paso 3: & Para todo: $i \in B$ y $j \in \mathrm{B}$ \\
\cline { 2 - 3 } & $\begin{array}{l}\text { Haga: Unión de nodos con todos los enlaces } \\
\text { posibles. }\end{array}$ \\
\hline Paso 4: & Si: $W_{i, j}$ es el menor de los existentes \\
\cline { 2 - 2 } Pasa 5: Enlace $i, j \in$ Árbol $A$ \\
\hline Paso 6: & $\begin{array}{l}\text { Problema principal: encuentre árbol de } \\
\text { expansión óptimo } A \text {, con enlaces } i, j \text { con } W_{i, j} \\
\text { más pequeños con Prim. }\end{array}$ \\
\hline \multicolumn{2}{|c|}{$\begin{array}{l}\text { Graficar árbol de expansión con los sitios } \\
\text { candidatos escogidos. }\end{array}$} \\
\hline \multicolumn{2}{|c|}{ Terminar } \\
\hline
\end{tabular}

\section{ANÁLISIS DE RESULTADOS}

En la Figura 2 se ha analizado un área rural en la que se han colocado 38 sitios candidatos de UDAPs así como 64 medidores inteligentes alrededor para proceder con el dimensionamiento.

Se considera que el porcentaje de cobertura de usuarios es del $100 \%$ y que se puede cubrir hasta

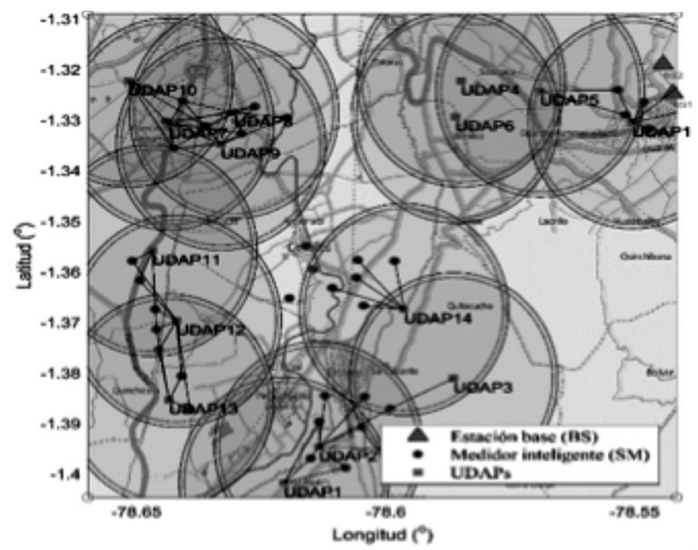

Figura 2. Escenario simulación.
300 medidores inteligentes por UDAP con una cobertura de dos $\mathrm{km}$, este caso es el que no se encuentra optimizado y se toma como base para la georreferenciación a los archivos SRTM de la NASA en su versión 3, en las gráficas por motivos estéticos se muestran solo 15 sitios candidatos.

La Figura 2 denota al mismo sistema georreferenciado mediante archivo OSM, se pueden denotar de mejor forma a las poblaciones involucradas.

Para la Figura 3 se denota como el algoritmo en su primera parte puede optimizar la cantidad de UDAPs, que deberán ser colocados cubriendo efectivamente a todos los usuarios/medidores y con ello minimizar el costo de implantación de tales sistemas.

La Figura 4 muestra el árbol de expansión óptimo que interconecta UDAPs, se puede notar que este

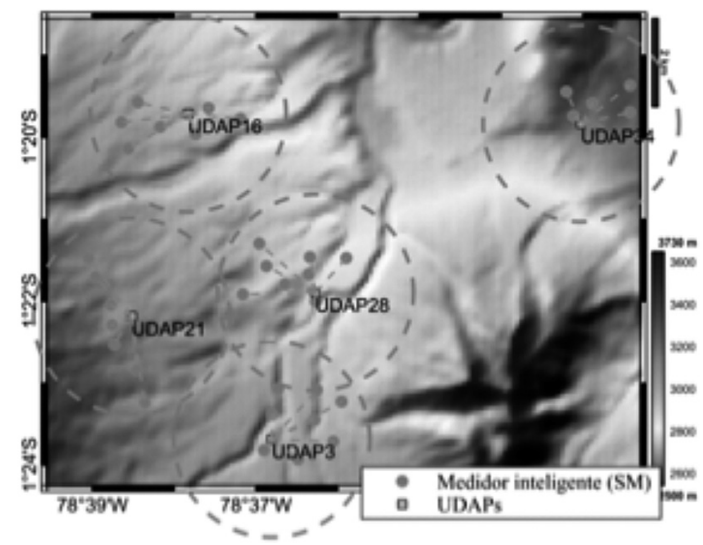

Figura 3. Optimización del número de UDAPs/costo.

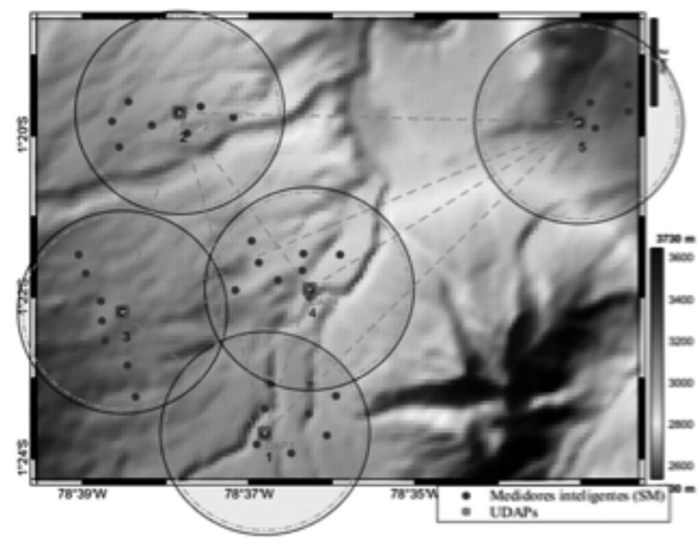

Figura 4. Enlaces factibles del árbol de expansión. 
algoritmo interconecta a todos los sitios candidatos, con todas las rutas posibles por las que pudiese pasar tal sistema.

La Figura 5 muestra el árbol de expansión que es óptimo interconectando a todos los UDAPs entre sí, siendo los caminos más óptimos para la infraestructura de comunicación a ser colocada. Se puede denotar que la ruta óptima sigue una forma en la que la distancia recorrida para interconectar todos los puntos efectivamente es la menor. Esto implica que cuando sea colocada la infraestructura de comunicación se podrá tener un costo menor para la implantación del sistema suponiendo ahorros para las compañías que están interesadas en la implantación de AMI.

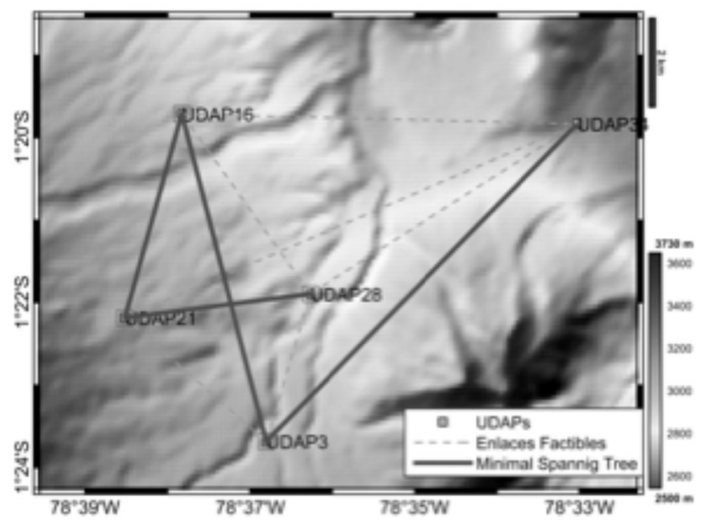

Figura 5. MST de enrutamiento UDAPs óptimo.

\section{CONCLUSIONES}

Los algoritmos planteados permiten resolver dos problemas respecto al despliegue de UDAPs en AMI, el primero es el dimensionamiento de la red, es decir minimizar el número de puntos a colocar de una serie de sitios candidatos planteados mediante escenarios iniciales, teniendo como restricciones la capacidad de cada punto de agregación así como la máxima distancia del radio de cobertura de cada tecnología, tal optimización permite minimizar el costo asociado a infraestructura de red e implementación, así como disminuir el tiempo para el despliegue al tener sitios georreferenciados que hayan eliminado la posibilidad de encontrar lagos, quebradas o sitio de difícil acceso.

El segundo problema es el enrutamiento de la infraestructura de comunicación para interconectar los UDAPs entre sí y luego poder alcanzar el centro de gestión, esto se realiza mediante el árbol de mínimo costo MST para interconectar todos los UDAP finalmente activos resueltos en el problema inicial mediante el uso de heurística para obtener la solución que es más cercana a la óptima e interconectar todos los puntos de agregación que están involucrados lo que le resultará muy útil a las compañías del sector eléctrico para implantar AMI.

Dentro de los planteamientos futuros se pretende incluir la variable de interferencia, por medio disponer un número de $K$ canales que estarían disponibles en cada tecnología inalámbrica, así también la posibilidad de que el enrutamiento sean planteado desde un problema de costo económico por enlace, debido a un costo elevado por instalación de un enlace, así como el costo de uso; esto es por costos relacionados a tuberías, obras civiles, y otros que resultan relevantes en el momento de colocar una infraestructura de comunicación.

\section{REFERENCIAS}

[1] J. Zhou, R.Q. Hu and Y. Qian. "Scalable Distributed Communication Architectures to Support Advanced Metering Infrastructure in Smart Grid". Parallel Distrib. Syst. IEEE Trans. Vol. 23 No 9, pp. 1632-1642. 2012. ISSN: 10459219. DOI:10.1109/ TPDS.2012.53.

[2] P. Li, S. Guo, S. Member and Z. Cheng. "Joint Optimization of Electricity and Communication Cost for Meter Data Collection in Smart Grid". Emerg. Top. Comput. IEEE Trans. Vol. $1 \mathrm{~N}^{\circ} 2$, pp. 297306. 2013. ISSN: 21686750 . DOI: $10.1109 /$ TETC.2013.2273890.

[3] F.J. Wu, Y.F. Kao and Y.C. Tseng. "From Wireless Sensor Networks Towards Cyber Physical Systems". Pervasive Mob. Comput. Vol. 7 No 4, pp. 397-413. 2011. ISSN: 15741192. DOI: 10.1016/j. pmcj.2011.03.003.

[4] V.C. Gungor, B.L. Bin Lu and G.P. Hancke. "Opportunities and Challenges of Wireless Sensor Networks in Smart Grid". Industrial Electronics IEEE Trans. Vol. $57 \mathrm{~N}^{\mathrm{o}} 10$, pp. 3557-3564. 2010. ISSN: 0278-0046. DOI: 10.1109/TIE.2009.2039455. 
[5] E. Inga, G. Arevalo and R. Hincapié. "Optimal Deployment of Cellular Networks for Advanced Measurement Infrastructure in Smart Grid". Commun. Comput. (COLCOM). IEEE Colomb. Conf., pp. 1-6. 2014. DOI: 10.1109/ColComCon.2014.6860421.

[6] X. Pan, X. Zhang, H. Yu and C. Zhang. "Study on Routing Protocol for WSNs Based on the Improved Prim Algorithm". 2009 International Conference on Wireless Communications and Signal Processing, WCSP, pp. 1-4. 2009. DOI: 10.1109/ WCSP.2009.5371396.

[7] K. Khamforoosh. "Clustered Balanced Minimum Spanning Tree for Routing and Energy Reduction in Wireless Sensor Networks". ISWTA 2011-2011 IEEE Symposium on Wireless Technology and Applications, pp. 56-59. 2011. DOI: 10.1109/ ISWTA.2011.6089553.

[8] J. Brown and J.Y. Khan. "Key Performance Aspects of an LTE FDD Based Smart Grid Communications Network". Comput. Commun. Vol. $36 \mathrm{~N}^{\circ}$ 5, pp. 551-561. 2013. ISSN: 01403664. DOI: 10.1016/j.comcom. 2012.12.007.

[9] B. Kalyanasundaram and M. Younis. "Using Mobile Data Collectors to Federate Clusters of Disjoint Sensor Network Segments". IEEE International Conference on Communications 2013. pp. 1496-1500. 2013. ISSN: 15503607. DOI: $10.1109 /$ ICC.2013.6654724.

[10] M. Khan, G. Pandurangan and V. S. A. Kumar. "Distributed Algorithms for Constructing Approximate Minimum Spanning Trees in Wireless Sensor Networks". IEEE Trans. Parallel Distrib. Syst. Vol. $20 \mathrm{~N}^{\circ}$ 1, pp. 124139. 2009. ISSN: 10459219 . DOI: $10.1109 /$ TPDS.2008.57.

[11] G. Souza, F.V. Mestrando, C. Lima, M. Castro and A. Sergio. "Optimal Positioning of GPRS Concentrators for Minimizing Node Hops in Smart Grids Considering Routing in Mesh Networks". 2013 IEEE PES Conf. Innov. Smart Grid Technol. ISGT Lat. Am., pp. 1-7. April, 2013. DOI: 10.1109/ ISGT-LA.2013.6554419.

[12] H.M. Ammari and S.K. Das. "Centralized and Clustered k-coverage Protocols for Wireless Sensor Networks". IEEE Trans.

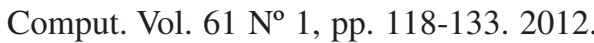
ISSN: 00189340. DOI: 10.1109/TC.2011.82.

[13] G. Barai and K. Raahemifar. "Optimization of Distributed Ccommunication Architectures in Advanced Metering Infrastructure of Smart Grid". Electrical and Computer Engineering (CCECE). IEEE 27th Canadian Conference on, pp. 1-6. 2014. DOI: 10.1109/ CCECE.2014.6901098.

[14] F. Aalamifar, G.N. Shirazi, M. Noori and L. Lampe. "Cost-efficient Data Aggregation Point Placement for Advanced Metering Infrastructure". 2014 IEEE International Conference on Smart Grid Communications (SmartGridComm), pp. 344-349. 2014. DOI: 10.1109/SmartGridComm. 2014.7007670.

[15] C. Wang, H. Liang, X. Geng and M. Zhu. "Multi-Sensor Fusion Method Using Kalman Filter to Improve Localization Accuracy Based on Android Smart Phone”. 2014 IEEE International Conference on Vehicular Electronics and Safety (ICVES), pp. 180-184. 2014. DOI: 10.1109/ICVES.2014.7063707.

[16] V. Fodor. "On the Gains of Deterministic Placement and Coordinated Activation in Sensor Networks". Global Telecommunications Conference. IEEE GLOBECOM 2008. IEEE, pp. 1-6. 2008. DOI: 10.1109/GLOCOM.2008. ECP.72.

[17] M.B. Mohammadi, R. Hooshmand and F.H. Fesharaki. "A New Approach for Optimal Placement of PMUs and Their Required Communication Infrastructure in Order to Minimize the Cost of the WAMS". IEEE Trans. Smart Grid. Vol. 7, Issuel, No 99, pp. 1-10. 2015. ISSN: 19493053. DOI: 10.1109/TSG.2015. 2404855.

[18] A. Peralta-Sevilla, E. Inga, R. Cumbal and R. Hincapié. "Optimum Deployment of FiWi Networks using Wireless Sensors based on Universal Data Aggregation Points". IEEE Colombian Conference on Communications and Computing, pp. 1-6. 2015.

[19] E. Inga, R. Hincapié, C. Suárez and G. Arévalo. "Shortest Path for Optimal Routing on Advanced Metering Infrastructure using Cellular Networks". IEEE Colombian Conference on Communications and Computing, pp. 1-6. 2015.

[20] N.M. Manousakis, G.N. Korres and P.S. Georgilakis. "Taxonomy of PMU 
placement methodologies". IEEE Trans. Power Syst. Vol. 27 No 2, pp. 1070-1077. 2012. ISSN: 08858950. DOI: 10.1109/ TPWRS.2011.2179816.

[21] B. Milosevic and M. Begovic. "Nondominated Sorting Genetic Algorithm for Optimal Phasor Measurement Placement”. 2003. IEEE Power Eng. Soc. Gen. Meet. Vol. 2 No 1, pp. 69-75. 2003. ISSN: 08858950. DOI: 10.1109/PES.2003.1270378.

[22] S. Dutta, D. Patra, H. Shankar and P. Alok Verma. "Development of GIS Tool for the Solution of Minimum Spanning Tree Problem using Prim's Algorithm". ISPRS - Int. Arch. Photogramm. Remote Sens. Spat. Inf. Sci. Vol. XL-8 N ${ }^{\circ}$ December, pp. 1105-1114. 2014. ISSN: 21949034. DOI: 10.5194/ isprsarchives-XL-8-1105-2014.

[23] Y. Choi, G. Pandurangan, M. Khan and V.S.A. Kumar. "Energy-Optimal Distributed Algorithms for Minimum Spanning Trees". IEEE J. Sel. Areas Commun. Vol. 27 N $^{\circ} 7$, pp. 1-9. 2009. ISSN: 07338716. DOI: 10.1109/JSAC. 2009.090924.

[24] AH. Mohammed, M.M. Mansour and M.A. Abido. "Application of Phasor
Measurement Units for fault location in SEC-EOA". IEEE International Energy Conference 2010, pp. 435-439. DOI: 10.1109/ ENERGYCON.2010.5771720.

[25] A. Daoudi, Y. Kerfi, I. Benelallam and E.H. Bouyakhf. "A Constraint Programming Approach for Coverage Optimization Problem in WSN". 2nd International Conference on Innovative Computing Technology. INTECH 2012, pp. 123-127. 2012. DOI: 10.1109 / INTECH.2012.6457791.

[26] A. Kocyigit. "An Optimal Network Dimensioning and Initial Energy Assignment Minimizing the Monetary Cost of a Heterogeneous WSN". Wireless Communication Systems, 2009. ISWCS 2009. 6th International Symposium on. pp. 517-521. 2009. DOI: 10.1109/ISWCS.2009.5285351.

[27] A. Raha, S. Maity, M.K. Naskar, O. Alfandi and D. Hogrefe. "An Optimal Sensor Deployment Scheme to Ensure Multi Level Coverage and Connectivity in Wireless Sensor Networks". Wireless Communications and Mobile Computing Conference (IWCMC). 8th International, pp. 299-304. 2012. DOI: 10.1109/IWCMC.2012.6314220. 